\title{
IAMJ
}

INTERNATIONAL

AYURVEDIC

MEDICAL JOURNAL

Research Article

ISSN: 2320-5091

Impact Factor: 6.719

\section{COMPARATIVE PHARMACEUTICO ANALYTICAL STUDY TO EVALUATE THE CONCEPT OF AVARTANA WITH SPECIAL REFERENCE TO 1, 10, 50, 100 AVARTITA MADHUKA TAILA}

\author{
Anagha. V. G' ${ }^{1}$, Vikram. $S^{2}$
}

PG Scholar ${ }^{1}$, Professor, HOD ${ }^{2}$

Dept. of PG Studies in Rasashastra and Bhaishajya Kalpana, Sri Sri College of Ayurvedic Science and Research, Bengaluru, Karnataka, India

\section{Corresponding Author: anaghavenugopal993@gmail.com}

\section{https://doi.org/10.46607/iamj0309072021}

(Published Online: July 2021)

Open Access

(C) International Ayurvedic Medical Journal, India 2021

Article Received: 17/06/2021 - Peer Reviewed: 24/06/2021 - Accepted for Publication: 25/06/2021

\section{Check for updates}

\section{ABSTRACT}

Avartana is a unique concept mentioned for Sneha Kalpana. The more the number of Avartana the more potent will be the formulation. "Shata Paka Madhuka Taila" is one such formulation that is explained in Charaka Chikitsa Vataraktha Adhikara. Samples were subjected to pharmaceutico - Analytical study to compare the probable changes that would have happened pharmaceutically \& analytically. Shata paka Madhuka Taila was prepared as per reference \& samples were Analysed. The change in the organoleptic characters of all the Avartita samples was noted. Consistency of taila to semisolid consistency of ghruta in proceeding Avartana was observed. A slight increase in specific gravity \& Refractive index were noted, decrease in acid value \& iodine value was observed. Rancidity test passed \& peroxide value was nil. slight acidic $\mathrm{pH}$ was noted. The presence of more bands with different Rf values was seen towards $50^{\text {th }}, 75^{\text {th }} \& 100^{\text {th }}$ Avartita samples on TLC. Pharmaceutical procedure reveals that concentration of the Active bio constituents of yastimadhu \& milk fat gets increased in each Avartana., And it was evident from organoleptic characters like conversion of liquid Consistency of taila to semisolid consistency of ghruta (on cooling) in proceeding Avartana. A slight increase in specific gravity \& Refractive index suggests more active constituents, decrease in acid value $\&$ iodine value suggests products are more stable $\&$ less chance of rancid on successive avartana. Rancidity \& peroxide value tells product are not rancid. $\mathrm{pH}$ changes indicate slight acidic 
nature $\&$ may be irritant due to high potency. TLC shows the presence of more bands towards $50^{\text {th }}, 75^{\text {th }} \& 100^{\text {th }}$ avarti samples shows more active constituents. Hence Avartana process helps for potentiating the formulation by adding active constituents.

Keywords: Sneha Kalpana, Avartana, Shata Paka Madhuka Taila.

\section{INTRODUCTION}

Ayurveda is the oldest traditional system of medicine in India. It is the eternal science that mainly deals with the knowledge of life. For the effective administration of active principles of the drug in a diseased condition, Different medicinal substances came into the form of Formulations by the repeated search of our Acharyas. Pancha vidha kashaya kalpanas i.e, Swarasa, Kalka, Kwatha, Hima, Phanta are the outcome of their thoughts. Acharya Charaka mentioned them as Pancha vidha kashaya kalpana for the first time ${ }^{1}$. Keeping this view in the mind several secondary preparations have been derived from these five basic preparations. And, Panchavidha Kashaya Kalpana having its lacunas, necessities which occurred in various areas like Dose, Shelf-life period, Route of administration, Availability, Easy way of administration of a drug, Palatability, Efficacy, to extract maximum active principles from drug various Upa kalpanas came into existence. Those are Sneha Kalpana, Sandhana kalpana, Vati kalpana, Avaleha kalpana, Rasayogas etc. Sneha Kalpana is the widely used dosage form for both External \& Internal uses. Sneha kalpana may be defined as "A pharmaceutical process to prepare oleaginous medicaments from the substances like Kalka, Kwatha \& Drava dravyas, in specific proportions by subjecting to a unique heating pattern and duration to fulfil certain pharmaceutical parameters, according to the need of therapeutics. This process ensures transformation of the active therapeutic properties of the ingredients to the solvents and hence to get fat-soluble, water-soluble or even the chemical constituents which are soluble in media like Kanji, Takra etc. ${ }^{2}$ Sneha preparations have better pharmacokinetic action in comparison to other dosage forms because of the lipoid nature of the biomembranes, as lipid-soluble substances readily permeate into the cells. The introduction of Sneha
Kalpana into Ayurvedic pharmaceutics may be the result of the above-mentioned facts. The rationality behind taking oil as a base is presumably to extract lipid-soluble active fractions from the ingredients into the oil and even this formulation holds its properties for a longer period when compared to primary preparations $^{3}$. Sneha is the fat or fatty material obtained from 2 yonies ie, Sthavara \& Jangama 4.4 types of Sneha Dravya are mentioned i.e, Sarpi, Majja, Vasa, Taila according to Ayurveda ${ }^{5}$. Taila Kalpana takes a Lion share. Because it is the only one such kalpana that is used for all 4 modes of drug administration like Pana, Abhyanga, Nasya \& Basthi, Through all the bodily routes of drug administration. Vata, the master controller of the body physiology when gets altered, is the Taila Kalpana which comes to help ${ }^{6}$.

Avartana is a unique concept mentioned for Sneha kalpana which prevails from the Samhita Kala. The concept of Dasha paki, Shata paki, Sahasra paki indicate the number of times, a process is repeated. The more the number of Avartana the more potent will be the formulation. Avartita Kalpana helps in improving therapeutic efficacy at low dosage, has quicker action, easy for administration and packaging. In our classics, the Concept of Avartana is explained for tailas like Bala Taila ${ }^{7}$ Ksheera bala taila ${ }^{8}$, Madhuka taila ${ }^{7}$ etc. Shata Paka Madhuka Taila is one such formulation which is explained in Charaka Chikitsa Vataraktha Adhikara where Avartana procedure is carried out ${ }^{7}$. Quicker relief from disease is the expectation of the hour. To achieve this expectation, the potentiation of our formulation is most essential. Avartita sneha is one such method said to impart more potency to the formulation. So, it is the need of the hour to do Avartana of taila kalpana for 
attaining above said points. That's why this study of "Shata paka Madhuka taila" has been taken up.

\section{OBJECTIVES}

1) To do Murchana Samskara to Tila taila.

2) To Prepare 1, 10, 50 Avartita Madhuka taila as per classical guidelines.

3) To Prepare 100 Avartita Madhuka Taila.

4) To carry out "Comparative Pharmaceutico Analytical study of 1, 10, 50, Avartita Madhuka taila with 100 Avartita Madhuka taila".

MATERIALS \& METHODS: Tila Taila Moorchana done as per reference of Bhaishajya Ratnavali Jwara rogadhikara ${ }^{9}$. Shatapaka Madhuka taila was prepared as per the reference of Charaka chikitsa vata raktha Adhikara. Paka Assessment was done as per Sharangadhara Samhitha Sneha Adhyaya $^{10}$. Ingredients for the preparation of Tila Taila Murchana \& Shata Paka Madhuka Taila was collected from Amrita kesari, Depot, Krishna raj market, Bangalore. Tila taila was collected from genuine source. The preparation of Tila Taila Murchana \& Shata Paka Madhuka taila was carried out in

Rasashastra \& Bhaishajya kalpana lab, Sri Sri College of Ayurveda, Bengaluru. Analysis was done in Sri Veda Tattva Pvt. Ltd. Bengaluru.

Tila Taila Murchana

Table 1: Ingredients of Tila Taila Murchana

\begin{tabular}{|c|c|c|c|c|}
\hline SI No & INGREDIENTS & BOTANICAL NAME & PROPORTION & QUANTITY TAKEN \\
\hline 1 & Tila Taila & Sesame oil & 1 Part & 1 Litre \\
\hline 2 & Manjista & Rubia cordifolia Linn & $1 / 16^{\text {th }}$ Part of Taila & $62.5 \mathrm{~g}$ \\
\hline 3 & Musta & Cyperus rotundus Linn & \multirow{10}{*}{$\begin{array}{l}\text { (From Musta } \\
\text { to vatangura) each one - }\end{array}$} & \multirow{10}{*}{$\begin{array}{l}\text { (From Musta } \\
\text { to vatangura) } \\
\text { each one - } \\
15 \mathrm{~g} \text { Each }\end{array}$} \\
\hline 4 & Hareetaki & Terminalia chebula Retz & & \\
\hline 5 & Vibhitaki & Terminalia belerica Roxb & & \\
\hline 6 & Amalaki & $\begin{array}{l}\text { Emblica } \\
\text { officinalis Gaertn.Syn }\end{array}$ & & \\
\hline 7 & $\begin{array}{l}\text { Nalika } \quad \text { (Raktha } \\
\text { Padma) }\end{array}$ & Nelumbo nucifera Gaertn. Syn. & & \\
\hline 8 & Hribera (Udichya) & Pavonia odorata willd. & & \\
\hline 9 & Haridra & Curcuma longa Linn & & \\
\hline 10 & Suchipushpa (Ketaki) & $\begin{array}{l}\text { Pandanus odoratissimus } \\
\text { Roxb.syn }\end{array}$ & & \\
\hline 11 & Lodra & Symplocos racemosa Roxb & & \\
\hline 12 & Vatagura & Ficus bengalensis Linn & & \\
\hline 13 & Jala & - & 4 Times to taila & 4 Litre \\
\hline
\end{tabular}

Preparation of Murchitha Tila taila: 1 Litre Tila taila was taken in a cleaned wide-mouthed stainlesssteel vessel \& placed over Mandagni. Once it was heated, slowly kalka dravyas (Total $212 \mathrm{~g}$ ) in 3 bolus form was added one by one $\&$ continuous stirring was done. It was followed by the addition of $4 \mathrm{~L}$ of Jala. Frequent stirring was done to allow Proper mixing of kalka dravya, Tila taila \& Jala. The procedure was not completed within 1 day. $1^{\text {st }}$-day paka done for 30 minutes \& $2^{\text {nd }}$ Sneha paka done for 2 hours 40 minutes.
Sneha paka was carried out till obtaining Taila Siddhi Lakshana \& Vessel was taken out from fire \& Taila was filtered through cloth when the kalka dravya was in lukewarm Condition to avoid much loss of Taila. obtained murchita Tila taila measured \& preserved. Precautions: kalka dravyas should be in fine form, Frequent stirring should be done to avoid sticking of kalka, Mandagni or madhyamagni was maintained throughout the procedure to avoid sticking of kalka. Observations: once kalka was added to the heated tila 
taila, within 15 seconds froth started to appear \& persist, along with fumes $\&$ peculiar sound was also appeared. froth occurred around double times of the oil $\&$ later subsided. followed by the addition of 4 times of jala \& stirring was carried out. After the addition of kalka \& jala to tila taila, the Whole mixture turned to reddish-brown colour. Initially stirring was less frequently done, later it was continuous. Later, boiling of whole contents started with fumes $(+++)$ of evaporation of water content. Further, the consistency of the whole mixture was changed to thicker compared to before $\&$ the quantity of mixture was also reduced. Later, Dark reddish coloured oil separated from kalka $\&$ started to boil. Less numbered bigger bubbles seen in the centre $\&$ more numbered smaller bubbles were seen on the sides. Later Murdu paka stage attained. On heating further, Madhyama paka stage started. kalka became soft in consistency \& made varti out of it. $k a l k a$ had the colour of brownish. Later, on keeping agni pareeksha, no crackling sound was heard. gandha, Varna, Rasa of murchitha tila taila was different from tila taila.

Table 2: Observations of Murchitha Tila taila

\begin{tabular}{|c|c|c|c|c|c|c|c|c|c|}
\hline Date & $\begin{array}{l}\text { Total } \\
\text { Hours }\end{array}$ & $\begin{array}{c}\text { Initial } \\
\text { Qty } \\
\text { of Tila } \\
\text { Taila } \\
\text { (In litre) }\end{array}$ & $\begin{array}{l}\text { Kalka } \\
\text { Qty } \\
\text { (In } \\
\text { Kilo) }\end{array}$ & $\begin{array}{c}\text { Qty of } \\
\text { Jala } \\
\text { needed } \\
\text { for } \\
\text { Kalka } \\
\text { Nirmana }\end{array}$ & $\begin{array}{c}\text { Drava } \\
\text { dravya } \\
\text { Jala }\end{array}$ & $\begin{array}{l}\text { Murchitha } \\
\text { Tila taila } \\
\text { obtained. } \\
\text { (In Litre) }\end{array}$ & $\begin{array}{c}\text { Loss / } \\
\text { Gain } \\
\text { (In } \\
\text { Litre) }\end{array}$ & $\begin{array}{c}\text { Loss / } \\
\text { Gain } \\
(\mathbf{I n} \\
\%)\end{array}$ & $\begin{array}{c}\text { Kalka } \\
\text { got } \\
\text { After } \\
\text { Filtration } \\
\text { (In Kilo) }\end{array}$ \\
\hline $\begin{array}{l}18^{\text {th }} \\
\text { Feb } \\
2020 \\
\text { to } \\
19^{\text {th }} \\
\text { Feb } \\
2020\end{array}$ & $\begin{array}{l}3 \mathrm{Hrs} \\
10 \mathrm{~min}\end{array}$ & $\begin{array}{l}1 \text { Litre } \\
\text { or } \\
1000 \mathrm{ml}\end{array}$ & $\begin{array}{l}0.212 \\
\mathrm{~kg} \\
\text { or } \\
212 \mathrm{~g}\end{array}$ & $\begin{array}{l}0.5 \text { Litre } \\
\text { or } \\
500 \mathrm{ml}\end{array}$ & $\begin{array}{l}4 \text { Litre } \\
\text { or } \\
4000 \mathrm{ml}\end{array}$ & $\begin{array}{l}0.98 \text { Litre } \\
\text { or } \\
980 \mathrm{ml}\end{array}$ & $\begin{array}{l}-20 \\
\mathrm{ml} \\
(20 \mathrm{ml} \\
\text { Loss) }\end{array}$ & $-2 \%$ & $\begin{array}{l}0.525 \mathrm{Kg} \\
\text { or } \\
525 \mathrm{~g}\end{array}$ \\
\hline
\end{tabular}

Table 3: Organoleptic characters of Tila Taila, Murchitha Tila taila

\begin{tabular}{|l|l|l|l|l|l|}
\hline SAMPLES & COLOUR & CONSISTENCY & APPEARANCE & ODOUR & TASTE \\
\hline Tila Taila & $\begin{array}{l}\text { Yellowish } \\
\text { Brown }\end{array}$ & oily & $\begin{array}{l}\text { Characteristic } \\
\text { oily odour }\end{array}$ & Oily & oily \\
\hline $\begin{array}{l}\text { Murchitha } \\
\text { Tila taila }\end{array}$ & $\begin{array}{l}\text { Dark } \\
\text { Reddish }\end{array}$ & $\begin{array}{l}\text { Oily (But thicker } \\
\text { than Tila taila) } \\
\text { Liquid }\end{array}$ & Oily & $\begin{array}{l}\text { Characteristic } \\
\text { oily smell }\end{array}$ & $\begin{array}{l}\text { Tiktha rasa }+ \\
\text { Slight madhura } \\
+ \text { oily }\end{array}$ \\
\hline
\end{tabular}


Table 4: Physicochemical Result of Tila taila, Murchitha Tila taila

\begin{tabular}{|l|l|l|}
\hline Name of Tests & Tila Taila & Murchitha Tila taila \\
\hline Specific Gravity & 0.9163 & 0.991 \\
\hline Refractive Index & 1.468 & 1.467 \\
\hline Acid Value & 4.17 & 4 \\
\hline Iodine Value & 110 & 23.9 \\
\hline Saponification value & 198 & 191.75 \\
\hline Total Fat content & 99.75 & 99.74 \\
\hline pH & 6.2 & 5.21 \\
\hline Peroxide value & Nil & Nil \\
\hline Rancidity & Pass & Pass \\
\hline
\end{tabular}

Table 5: TLC Results: Rf values \& colors of the chromatogram of various samples viewed at $254 \mathrm{~nm}$

\begin{tabular}{|l|l|l|}
\hline SI No & SAMPLE & TLC RESULTS - Rf Values \\
\hline 1 & Murchitha Tila Taila & 0.86 Blue \\
& & 0.92 Purple \\
& & 0.97 Dark blue \\
\hline
\end{tabular}

\section{PREPARATION OF SHATAPAKA MADHUKA TAILA}

Table 6: Showing Preparation of Shatapaka Madhuka Taila

\begin{tabular}{|c|c|c|}
\hline $\begin{array}{l}\text { Practical } \\
\text { Number }\end{array}$ & $\begin{array}{l}\text { Shata Paka Madhuka } \\
\text { Taila }\end{array}$ & $\begin{array}{l}\text { It Includes Preparation starting from } 1^{\text {st }} \text { Avartana of Madhuka taila to } 100^{\text {th }} \\
\text { Avartana of Madhuka taila. }\end{array}$ \\
\hline $1^{\mathrm{st}}$ & $\begin{array}{l}\text { Preparation of } 1^{\text {st }} \text { Avartana } \\
\text { of Madhuka taila }\end{array}$ & $\begin{array}{l}\text { Sneha Paka was carried out till attaining Sneha siddhi Lakshana by taking } \\
\text { Murchitha tila taila as base }(500 \mathrm{ml}) \text {, Yastimadhu as kalka }(31 \mathrm{~g}) \text {, Drava } \\
\text { dravyas as ksheera }\left(2 \text { Litre) \& Jala }(2 \mathrm{Litre}) \rightarrow \text { Obtained } 1^{\text {st }} \text { Avartita Madhuka }\right. \\
\text { Taila was Measured \& preserved. }(540 \mathrm{ml})\end{array}$ \\
\hline $2^{\text {nd }}$ & $\begin{array}{l}\text { Preparation of } 2^{\text {nd }} \text { Avartana } \\
\text { Madhuka taila }\end{array}$ & $\begin{array}{l}\text { Sneha paka was carried out by taking obtained quantity of } 1^{\text {st }} \text { Avartita Madhuka } \\
\text { taila }(540 \mathrm{ml}) \text { as base, Yastimadhu kalka }(33 \mathrm{~g}) \text {, Ksheera. } \\
(2160 \mathrm{ml}) \& \text { jala }(2160 \mathrm{ml}) \rightarrow \text { Obtained } 2^{\text {nd }} \text { Avartita Madhuka taila was } \\
\text { measured. }\end{array}$ \\
\hline $\begin{array}{l}3^{\text {rd }} \text { to } \\
100^{\text {th }}\end{array}$ & $\begin{array}{l}\text { Preparation of } 3^{\text {rd }} \text { Avartana } \\
\text { Madhuka taila to } 100^{\text {th }} \\
\text { Avartana of Madhuka taila }\end{array}$ & $\begin{array}{l}\text { As per the measured quantity of previously Avartita Madhuka taila, } \\
\text { Yastimadhu kalka, ksheera \& jala were added proportionally \& Taila paka was } \\
\text { carried out. The process was carried out till } 100 \text { Avarti Attained. }\end{array}$ \\
\hline
\end{tabular}


Table 7: Organoleptic characters of Different Avartita Preparations of Madhuka Taila

\begin{tabular}{|c|c|c|c|c|c|c|c|c|}
\hline $\begin{array}{l}\text { Name of the } \\
\text { Preparation }\end{array}$ & $\begin{array}{l}\text { Start.Date/Time \& } \\
\text { End. Date/Time }\end{array}$ & $\begin{array}{l}\text { Paka } \\
\text { Kala }\end{array}$ & $\begin{array}{l}\text { Taila } \\
\text { Qty }(\mathrm{ml})\end{array}$ & $\begin{array}{l}\text { Kalka } \\
\text { Qty }(g)\end{array}$ & $\begin{array}{l}\text { Ksheera } \\
\& \text { jala } \\
\text { Qty }(\mathrm{ml})\end{array}$ & $\begin{array}{l}\text { Taila } \\
\text { obtained }\end{array}$ & $\begin{array}{l}\text { Kalka } \\
\text { Obtained }\end{array}$ & $\begin{array}{l}\text { Gain/Loss } \\
(\mathrm{ml} \& \%)\end{array}$ \\
\hline $\begin{array}{l}1^{\text {st }} \text { Avartita } \\
\text { Madhuka } \\
\text { Taila }\end{array}$ & $\begin{array}{l}19-02-2020(3.30 \\
\text { pm to } 4 \mathrm{pm}) \\
20-02-2020(11 \\
\text { am to } 12.30 \mathrm{pm})\end{array}$ & $2 \mathrm{~h}$ & $500 \mathrm{ml}$ & $31 \mathrm{~g}$ & $\begin{array}{l}2000 \mathrm{ml} \\
\text { each }\end{array}$ & $540 \mathrm{ml}$ & $329 g$ & $\begin{array}{l}+40 \mathrm{ml} \\
(8 \%)\end{array}$ \\
\hline $\begin{array}{l}10^{\text {th }} \text { Avartita } \\
\text { Madhuka } \\
\text { Taila }\end{array}$ & $\begin{array}{l}28-02-2020(3.35 \mathrm{pm} \\
\text { to } 5.40 \mathrm{pm}) \\
29-02-2020(9.15 \mathrm{am} \\
\text { to } 9.40 \mathrm{pm}\end{array}$ & $\begin{array}{l}2 \mathrm{~h} \\
35 \\
\min \end{array}$ & $740 \mathrm{ml}$ & $46 \mathrm{~g}$ & $\begin{array}{l}2960 \mathrm{ml} \\
\text { each }\end{array}$ & $790 \mathrm{ml}$ & $319 \mathrm{~g}$ & $\begin{array}{l}+50 \mathrm{ml} \\
(6.75 \%)\end{array}$ \\
\hline $\begin{array}{l}50^{\text {th }} \text { Avartita } \\
\text { Madhuka } \\
\text { Taila }\end{array}$ & $\begin{array}{l}13-06-2020(5.10 \\
\text { pm to } 12.20 \mathrm{am}) \\
14-06-2020(7.45 \\
\text { am to } 2 \mathrm{pm})\end{array}$ & $\begin{array}{l}13 \mathrm{~h} \\
25 \\
\min \end{array}$ & $4540 \mathrm{ml}$ & $283 \mathrm{~g}$ & $\begin{array}{l}18160 \mathrm{ml} \\
\text { each }\end{array}$ & $4700 \mathrm{ml}$ & $3200 \mathrm{~g}$ & $\begin{array}{c}+160 \mathrm{ml} \\
(3.5 \%)\end{array}$ \\
\hline $\begin{array}{l}100^{\text {th }} \\
\text { Avartita } \\
\text { Madhuka } \\
\text { Taila }\end{array}$ & $\begin{array}{l}06-08-2020(8.45 \\
\text { am to } 11.05 \mathrm{pm}) \\
07-08-2020 \\
(10.40 \mathrm{am} \\
\text { to } 5.30 \mathrm{pm})\end{array}$ & $\begin{array}{l}21 \mathrm{~h} \\
10 \\
\min \end{array}$ & $7320 \mathrm{ml}$ & $457 \mathrm{~g}$ & $\begin{array}{l}29280 \mathrm{ml} \\
\text { each }\end{array}$ & $7440 \mathrm{ml}$ & $4705 \mathrm{~g}$ & $\begin{array}{l}+120 \mathrm{ml} \\
(1.63 \%)\end{array}$ \\
\hline
\end{tabular}

Table 8: Observations of Different Avarti preparations of Madhuka Taila

\begin{tabular}{|c|c|c|c|c|c|}
\hline Preparation & Colour & Appearance & Smell & Taste & Consistency \\
\hline $\begin{array}{l}1^{\text {st }} \text { Avartita } \\
\text { Madhuka } \\
\text { Taila }\end{array}$ & $\begin{array}{l}\text { Dark reddish } \\
\text { (colour Lighter } \\
\text { than Murchitha } \\
\text { tila taila }\end{array}$ & $\begin{array}{l}\text { Oily, Liquid (Thicker } \\
\text { than murchitha tila } \\
\text { taila) }\end{array}$ & $\begin{array}{l}\text { Characteristic } \\
\text { oily smell }\end{array}$ & Oily, Tiktha & $\begin{array}{l}\text { Liquid, oily } \\
\text { (Thicker in } \\
\text { consistency }\end{array}$ \\
\hline $\begin{array}{l}1^{\text {th }} \text { Avartita } \\
\text { Madhuka } \\
\text { Taila }\end{array}$ & $\begin{array}{l}\text { Yellowish } \\
\text { Brown }\end{array}$ & Ghee on cooling & $\begin{array}{l}\text { Smell of } \\
\text { yastimadhu+ } \\
\text { Ghee smell }\end{array}$ & $\begin{array}{l}\text { More madhura+ } \\
\text { Ghee taste }\end{array}$ & $\begin{array}{l}\text { Semisolid more, } \\
\text { ghee like on } \\
\text { cooling }\end{array}$ \\
\hline $\begin{array}{l}\mathbf{5 0}^{\text {th }} \text { Avartita } \\
\text { Madhuka } \\
\text { Taila }\end{array}$ & $\begin{array}{l}\text { More yellowish }+ \\
\text { slight brownish } \\
\text { Coloured } \\
\text { Ghee only }\end{array}$ & Ghee on cooling & $\begin{array}{l}\text { Smell of } \\
\text { yasti madhu+ } \\
\text { ghee }\end{array}$ & $\begin{array}{l}\text { More madhura } \\
\text { tiktha less + ghee`s } \\
\text { taste more }\end{array}$ & $\begin{array}{l}\text { Fully Turned to } \\
\text { ghee on cooling }\end{array}$ \\
\hline $\begin{array}{l}100^{\text {th }} \\
\text { Avartita } \\
\text { Madhuka } \\
\text { Taila }\end{array}$ & $\begin{array}{l}\text { Yellowish } \\
\text { brownish } \\
\text { Coloured } \\
\text { Ghee only }\end{array}$ & Ghee on cooling & $\begin{array}{l}\text { Smell of } \\
\text { yasti madhu+ } \\
\text { ghee }\end{array}$ & $\begin{array}{l}\text { Madhura rasa + } \\
\text { slight tiktha rasa - } \\
\text { both equal }\end{array}$ & $\begin{array}{l}\text { Fully Turned to } \\
\text { ghee on cooling }\end{array}$ \\
\hline
\end{tabular}

Common Procedure: Previously Avartita Madhuka taila was taken in a wide mouthed stainless-steel vessel. It was kept over gas stove \& fire was Ignited \& allowed to heat over Mandagni. once it was Heated, Drava dravyas (Ksheera \& Jala) 4 times to taila was added one after another $\&$ stirring was done. Followed by addition of Yastimadhu Kalka (1/16 ${ }^{\text {th }}$ part of taila)
$\&$ stirring was done. The procedure was not completed within a day. The next day also done until obtaining siddhi Lakshana \& Vessel was taken out from fire \& obtained taila was filtered through cloth when the kalka dravya was in Lukewarm condition to avoid much loss of Taila. Obtained Avartita Madhuka taila was measured \& preserved. Precautions: kalka 
dravya should be in Fine form. frequent stirring with mandagni or madhyamagni should be maintained throughout the procedure to avoid sticking of kalka. After the $1^{\text {st }}$ day Paka of taila for few hours, should be kept closed with a lid (after it cools down) to avoid falling off any impurities. stages of paka observed very carefully. filtration of taila has to be done in the hot stage to avoid loss. obtained Avartita Madhuka taila measured \& preserved.

Table 9: Physico - chemical Result of Different Avartita Madhuka Taila Samples

\begin{tabular}{|c|c|c|c|c|c|c|}
\hline $\begin{array}{l}\text { Name of } \\
\text { Tests }\end{array}$ & $\begin{array}{l}1^{\text {st }} \text { Avartita } \\
\text { Madhuka Taila }\end{array}$ & $\begin{array}{l}\text { 10 }^{\text {th }} \text { Avartita } \\
\text { Madhuka Taila }\end{array}$ & $\begin{array}{l}25^{\text {th }} \text { Avartita } \\
\text { Madhuka } \\
\text { Taila }\end{array}$ & $\begin{array}{l}\mathbf{5 0}^{\text {th }} \text { Avartita } \\
\text { Madhuka } \\
\text { Taila }\end{array}$ & $\begin{array}{l}\text { 75 }^{\text {th }} \text { Avartita } \\
\text { Madhuka } \\
\text { Taila }\end{array}$ & $\begin{array}{l}\text { 100 }^{\text {th }} \text { Avartita } \\
\text { Madhuka } \\
\text { Taila }\end{array}$ \\
\hline $\begin{array}{l}\text { Specific } \\
\text { Gravity }\end{array}$ & 0.994 & 0.991 & 0.991 & 0.994 & 0.990 & 0.991 \\
\hline Refractive index & 1.466 & 1.466 & 1.469 & 1.469 & 1.469 & 1.468 \\
\hline $\begin{array}{l}\text { Acid } \\
\text { value }\end{array}$ & 4.12 & 3.32 & 3.25 & 3.1 & 3.19 & 3.4 \\
\hline $\begin{array}{l}\text { Iodine } \\
\text { value }\end{array}$ & 24.1 & 24.8 & 23.89 & 24.3 & 24.89 & 24.8 \\
\hline $\begin{array}{l}\text { Saponification } \\
\text { value }\end{array}$ & 191.32 & 191.71 & 190.62 & 191.99 & 191.62 & 191.75 \\
\hline $\begin{array}{l}\text { Total Fat } \\
\text { content }\end{array}$ & 99.64 & 99.79 & 99.70 & 99.79 & 99.72 & 99.71 \\
\hline $\mathrm{pH}$ & 5.32 & 5.89 & 5.62 & 5.26 & 5.91 & 5.83 \\
\hline $\begin{array}{l}\text { Peroxide } \\
\text { value }\end{array}$ & Nil & Nil & Nil & Nil & Nil & Nil \\
\hline Rancidity & Pass & Pass & Pass & Pass & Pass & Pass \\
\hline
\end{tabular}

Table 10: TLC Results - Rf values \& colours of the chromatogram of various samples viewed at $254 \mathrm{~nm}$

\begin{tabular}{|l|l|l|}
\hline SI No & Samples & TLC Results \\
\hline $\mathbf{1}$ & $\mathbf{1}^{\text {st }}$ Avartita & 0.84 Red \\
& Madhuka Taila & 0.89 Purple \\
& & 0.95 Dark blue \\
\hline $\mathbf{2}$ & $\mathbf{1 0}^{\text {th }}$ Avartita & 0.84 Red \\
& Madhuka Taila & 0.90 Purple \\
& & 0.93 Dark blue \\
\hline $\mathbf{3}$ & $\mathbf{2 5}^{\text {th }}$ Avartita & 0.85 Red \\
& Madhuka Taila & 0.90 Purple \\
& & 0.95 Dark blue \\
\hline $\mathbf{4}$ & $\mathbf{5 0}^{\text {th }}$ Avartita & 0.56 Green \\
& Madhuka Taila & 0.70 Red \\
& & 0.77 Blue \\
\hline $\mathbf{5}$ & & 0.87 Green \\
\hline & $\mathbf{7 5}^{\text {th }}$ Avartita & 0.70 Blue \\
& Madhuka Taila & 0.88 Red \\
\hline $\mathbf{6}$ & & 0.91 Purple \\
& & 0.97 Dark blue \\
\hline & $\mathbf{1 0 0}^{\text {th }}$ Avartita & 0.56 Green \\
& Madhuka Taila & 0.70 Red \\
& & 0.75 Blue \\
& & 0.87 green \\
\hline
\end{tabular}




\section{DISCUSSION}

To understand the concept of Avartana, Shata paka ie, 100 paka of Madhuka taila was carried out. Murchitha tila taila was taken for the preparation of Shata paka Madhuka taila. During Murchana of Tila taila, Jala was added 4 times to the quantity of taila for the samyak paka (proper extraction) of the kalka even though not in yoga. After addition of kalka, suddenly froath \& fumes with peculiar sound appears on oil, due to moisture content. oily consistency of taila was reduced after the addition of jala into the taila, mostly because of less viscous nature of jala. During the boiling of oil, due to water evaporation, less numbered bigger bubbles seen in the centre \& more numbered smaller bubbles seen on the sides to appear. froth started appearing in the surface of taila suggestive of samyak paka lakshana of taila. Because taila contains unsaturated fatty acids which on heating continuous oxidation happens, lower fatty acids generated leads to bubbling. on completion of paka due to the conversion from unsaturated fatty acids to saturated fatty acids. In mrudu paka stage ${ }^{6}$, varti of kalka couldn't be able to prepare \& crackling sound heard on agni due to the moisture content in it. In Madhyama paka stage ${ }^{6}$ crackling sound was absent by putting taila \& kalka on fire, suggests devoid of moisture. spillage \& evaporation of taila during heating \& absorption of taila by cloth during filtration were the reasons for the loss of $20 \mathrm{ml}$ of Murchitha tila taila. characteristic odour, slight Madhura \& Tiktha rasa with characteristic oily taste \& Dark reddish varna, Thicker in the consistency of Murchitha tila taila suggestive of entry of active constituents of kalka to taila. In the preparation of Shata paka Madhuka Taila, Jala was added 4 times to the quantity of taila for samyak paka of the kalka (proper extraction) as ksheera was the drava dravya ${ }^{7}$. The initial addition of yastimadhu kalka to oil was not followed due to chances of burning in direct contact with hot taila, so it was added after drava dravyas. ie, ksheera \& jala. Bhaishajya Ratnavali reference also supports to this order of addition. In stage of boiling of milk, the temperature in different avartita samples ie, $1^{\text {st }}, 10^{\text {th }}, 50^{\text {th }} \& 100^{\text {th }}$ was $99.5^{0} \mathrm{c}, 99.4^{0} \mathrm{c}, 99.6^{0} \mathrm{c}, 99.5^{\circ} \mathrm{c}$ respectively. As the boiling point of milk is $95^{\circ} \mathrm{c}$, so all reached that level. continues stirring was not required as the whole mixture was liquid, non-thick \& non-sticky. In the stage of mixing, continuous stirring was essential because the mixture was thick, sticky in consistency. In the initial number of Avarti preparations, mixture colour was pinkish red due to a combination of reddish taila, yellowish-brown yastimadhu kalka \& whitish ksheera. Later number of Avartita tila had light yellowish-brown, further onwards brownish coloured taila was mostly due to the addition of yastimadhu kalka \& ksheera in each Avarti. In further stages of separation of oil \& boiling of oil, temperature found was more, as the water evaporated more. In stage of paka lakshana, up to $6^{\text {th }}$ Avartana, phena udgama was seen, later onwards slowly phena shanti observed due to conversion to ghee. For attaining vishesha guna sanchaya to taila as per sharangadhara, each taila paka was not completed within a day. if we keep these preparations in ratri, it will help for the vishesha guna sanchaya (for the entry of active constituents into sneha). so, 100 nights were kept as it is in the night after few hours of paka in the daytime. Reasons for colour change of oil from dark reddish, light reddish, yellowish, yellowish-brown successively in Avarti due to addition of yastimadhu, ksheera, continuous agni paka \& also due to milk fat conversion to ghee. consistency changed from liquid, oily to partial semisolid, further semi solidness increased \& converted to ghee form due to conversion of milk fat. Murchitha tila taila had an oily taste, slight tiktha, Madhura rasa. $1^{\text {st }}$ Avarti had oily, tiktha, on successive avarti madhura rasa also added. on $7^{\text {th }}$ slightly more madhura \& less tiktha rasa \& mixed taste of ghruta \& taila. further onwards more madhura, less tiktha, ghee taste more, later more tiktha, less madhura, ghee taste more, further both madhura \& tiktha rasa almost equal with ghee taste towards shata paka. Reason for taste change due to addition of the yastimadhu, ksheera (Milk fat conversion into Ghee) \& agni paka also. In the case of odour, murchitha tila taila had a characteristic smell. Further onwards yastimadhu smell \& ghruta smell was appreciated. later onwards same odour with more intensity was appreciated. From 
$1^{\text {st }}$ Avarti to $50^{\text {th }}$ Avarti 9.4 times of increment \& from $50^{\text {th }}$ Avarti to $100^{\text {th }}$ Avarti 6.4 times increment of total quantity was there. Reason for more yield was milk fat conversion to ghruta form. As compared to gain, the loss was less because of the addition of ingredients in each avartana. Specific Gravity results suggest that compared to Tila tila, all samples specific gravity got increased suggestive of the presence of active Constituents. Marginal increase in the value of the refractive index in successive avartana due to additional phytoconstituents. After murchana acid value decreases also on successive avartana acid value decreases. Tila taila had a high acid value suggests higher fatty acids content $\&$ thereby faster is the rancidification as compared to others. So, on successive Avartana Acid value decreases. Ie, Product will be more stable towards successive Avarti. The iodine values of all the samples are very less compared to Tila taila. So, all samples are less reactive, stable, and less susceptible to oxidation \& rancidification. There were not many changes in the fat value \& saponification value of samples. $\mathrm{pH}$ value suggests that compared to tila taila all samples are a little acidic. Mostly due to the addition of murchana drugs \& also due to the addition of milk (milk also slightly acidic). Samples May slight irritant in nature as it is with potency. $\mathrm{pH}$ variation due to continuous heat exposure. Peroxide values are zero for all the samples So none of the samples got rancid. Rancidity showing as all are pass means products are not rancid.

TLC results show that murchitha tila taila having 3 bands \& the blue band in murchitha tila taila is replaced by the red band in $1^{\text {st }}, 10^{\text {th }} \& 25^{\text {th }}$ Avartita Madhuka taila mostly due to the active constituents by the addition of yastimadhu \& milk. $1^{\text {st }}, 10^{\text {th }}, 25^{\text {th }}$ Avartita Madhuka taila having similar 3 bands with different $\mathrm{Rf}$ values. $50^{\text {th }}, 75^{\text {th }} \& 100^{\text {th }}$ Avartita Madhuka taila shows different 4 bands with different $\mathrm{Rf}$ values. The reason for the presence of more bands in the $50^{\text {th }}, 75^{\text {th }} \& 100^{\text {th }}$ Avartita Madhuka taila samples might be because of the presence of more active principles.

\section{CONCLUSION}

To evaluate the concept of Avartana a study was undertaken to prepare 1, 10, 50,100 Avartita madhuka taila \& carry out a comparative pharmaceutico analytical study. The pharmaceutical procedure reveals that the concentration of the active bio constituents of yastimadhu \& milk fat gets increased in each avartana. There was a conversion of liquid consistency of taila to semisolid consistency of ghruta (on cooling) in proceeding avartana due to the conversion of added milk fat to ghee \& change in colour, consistency, odour, taste, appearance also noted. Analytical study reveals that increase in specific gravity \& Refractive index suggests more active constituents, decrease in acid value $\&$ iodine value suggests product are more stable \& less chance of rancid on successive avartana. Rancidity \& peroxide value tells product are not rancid. No significant changes were seen in saponification \& fat value. $\mathrm{pH}$ changes indicate slight acidic nature \& may be irritant due to high potency. TLC shows the presence of more bands towards $50^{\text {th }}, 75^{\text {th }} \& 100^{\text {th }}$ avarti samples shows more active constituent. It can be concluded that pharmaceutically \& Analytically concentration of Bioconstituents are more present in successive Avartana. Thus, Avartana process helps for potentiating the formulation by adding active constituents.

\section{REFERENCES}

1. Agnivesha, Charaka Samhita of Acharya Charaka with Ayurveda Dipika Commentary by Chakrapani Datta, Dridhabala krit, edited by Vaidya Yadavji Trikamji Acharya. Sutra sthana. Su. 30, ver.7.1 ${ }^{\text {st }}$ edition reprint, Varanasi: Chaukhambha Surbharathi Prakashan; 2011.p.31.

2. M. S krishnamurthy. A comparative pharmaco chemical study of 1 -7- 50 avartita Ksheera bala taila prepared by atibala and their clinical efficacy in the management of sandhigata vata (MD Dissertation). Jamnagar: IPGT\& RA; 2002.p.14

3. Anarnath Bekal Soumya. A Critical comparative study on the importance of Kaalamaryada in Sneha paka w.s.r to Nirgundi Taila (MD Dissertation). SDM Udupi: Rajiv Gandhi University of Health Science;2010 [cited on 2020 June 24].p.1 Available from: 
http://52.172.27.147:8080/jspui/bitstream/123456789/ 5142/1/Bekal\%20Soumya\%20Amarnath.pdf

4. Agnivesha, Charaka samhitha of Acharya Charaka with Ayurveda Dipika commentary by chakrapani Datta, Dridhabala krit, edited by Vaidya Yadavji Trikamji Acharya. Sutra sthana. Su.13, ver. 9-11. $1^{\text {st }}$ edition reprint, Varanasi: Chaukhambha Surbharathi Prakashan; 2011.p.81.

5. Agnivesha, Charaka Samhitha of Acharya Charaka with Ayurveda Dipika commentary by chakrapani Datta, Dridhabala krit, edited by Vaidya Yadavji Trikamji Acharya. Sutra sthana. Su.13, ver. 12. $1^{\text {st }}$ edition reprint, Varanasi: Chaukhambha Surbharathi Prakashan; 2011.p.81.

6. M.S krishnamurthy. A comparative pharmacochemical study of 1 -7- 50 avartita.Ksheera bala taila prepared by atibala and their clinical efficacy in the management of sandhigata vata (MD Dissertation). Jamnagar: IPGT\& RA; 2002. p.7

7. Agnivesha, Charaka Samhitha of Acharya Charaka with Ayurveda Dipika commentary by chakrapani datta, Dridhabala krit, edited by Vaidya Yadavji Trikamji Acharya. chikitsa sthana. chi.29, ver.117$118.1^{\text {st }}$ edition reprint, Varanasi: Chaukhambha Surbharathi Prakashan; 2011. p.632.

8. Vagbhata, Astanga Hrudayam of Acharya Vagbhata with Sarvanga sundara of Arunadatta and Ayurveda
Rasayana of Hemadri, edited by Hari sadashiva sastri paradakara bhishagacarya. Chikitsa sthana. chi.22, ver.45-46. $1^{\text {st }}$ edition reprint, Varanasi: Chaukhamba Krishnadas Academy; 2006. p.732.

9. Kaviraj Govinda Das Sen, Bhaishajya Ratnavali with siddhiprada hindi commentary by Siddhi Nandan Mishra. Chapter.1, ver.1264-1295. $1^{\text {st }}$ Ed, varanasi: Chowkhabha samskrutha samsthana; 2005.p.206

10. Sharangadhara acharya, Sharagadhara samhitha of Sharangadharacharya with the commentary Adhamalla`s Dipika \& Kasirama Vaidya`s Gudartha Dipika. Edited by Shastry Parashuram. Madhyama khanda. chapter. 9, ver.11. Varanasi: Chaukhambha surabharati prakashan; 2013.p.213

\section{Source of Support: Nil \\ Conflict of Interest: None Declared}

How to cite this URL: Anagha. V. G \& Vikram. S: Comparative Pharmaceutico Analytical Study To Evaluate The concept Of Avartana With Special Reference To 1, 10, 50, 100 Avartita Madhuka Taila. International Ayurvedic Medical Journal \{online\} 2021 \{cited July 2021\} Available from: http://www.iamj.in/posts/images/upload/1334_1343.pdf 